Reprod. Nutr. Dévelop., 1983, 23 (5), 889-898.

\title{
Quelques aspects de l'évolution de l'ovaire de Typhlonectes compressicaudus (Duméril et Bibron, 1841), Batracien Apode vivipare. - Etude quantitative et histochimique des corps jaunes
}

\section{J. M. EXBRAYAT, Germaine COLLENOT \\ avec la collaboration technique de F. ALLIZARD et M. T. LAURENT}

\author{
Laboratoire de Biologie générale de la Faculté Catholique des \\ Sciences de Lyon et Laboratoire d'Etude du Développement post. \\ embryonnaire des Vertébrés inférieurs, de L'Ecole Pratique des \\ Hautes Etudes, 25, rue du Plat, 69288 Lyon Cedex 1. \\ Laboratoire d'Anatomie comparée, Université Paris VII, \\ 2, place Jussieu, 75221 Paris Cedex 05.
}

Summary. Some aspects of the development of the ovary of Typhlonectes compressicaudus (Duméril and Bibron, 1841), a viviparous apodan amphibian. - A quantitative and histochemical study of the corpora lutea.

Structures similar to the corpora lutea of viviparous Urodela and Anura have been observed in Typhlonectes compressicaudus, a viviparous apodan amphibian. The present paper reports a morphological and biometrical study of these structures, showing their parallel development with that of embryos in the uterus. $3 \beta$-HSDH activity and marked lipids were detected by histochemical procedures. We obtained positive results in the granulosa and theca of vitellogenic oocytes, positive results in the cells of supposed corpora lutea only after ovulatory laying, and no enzymatic activity in atretic foolicles from unlaid oocytes. This study shows that gravid females of $T$. compressicaudus have corpora lutea and that their development is closely related to pregnancy.

\section{Introduction.}

Typhlonectes compressicaudus est un Amphibien Apode vivipare qui vit en Guyane Française dans les savanes immergées. Au cours de diverses missions réalisées grâce à la Fondation Singer-Polignac, nous avons pu collecter un matériel important qui nous a permis de déterminer le cycle sexuel de cet animal (Exbrayat, Delsol et Flatin, 1981 ; Exbrayat et Sentis, 1982 ; Exbrayat, 1983).

Le cycle de Typhlonectes compressicaudus est étroitement lié à l'alternance des saisons sèches (juillet-août à décembre-janvier) et humide (décembre-janvier à juillet-août). La croissance des ovocytes a lieu en avril-mai, chez toutes les femelles adultes, gestantes ou non. Entre octobre et décembre, la vitellogenèse affecte 80 à $100 \%$ des femelles adultes. La ponte ovulaire intervient au début de 
la saison humide, entre février et avril. La ponte ovulaire et la gestation n'affectent que la moitié environ des femelles adultes. La période de gestation s'étale jusqu'en juillet pour les premières mises bas et en septembre ou même octobre pour les dernières. Les ovocytes pondus donnent lieu à la formation de structures semblables au corps jaunes observés chez d'autres espèces, Apodes (Wake, 1968, 1977) ou Amphibiens vivipares ou ovovivipares (Salamandra atra : Vilter et Vilter, 1960, 1964 ; Salamandra salamandra : Joly, 1964, 1965 ; Joly et Picheral, 1972 ; Nectophrynoides occidentalis : Lamotte et Rey, 1954 ; Lamotte, Rey et Vogeli, 1964 ; Ozon et Xavier, 1968 ; Xavier, Zuber-Vogeli, Le Quang Trong, 1970 ; Xavier et Ozon, 1971). Comme chez la plupart des espèces, les ovocytes en vitellogenèse non pondus donnent lieu à la formation de follicules atrétiques.

Le but de ce travail est de préciser l'évolution de l'ovaire au cours de la gestation en mettant particulièrement l'accent sur l'existence et le devenir des corps jaunes ainsi que sur leur rôle endocrinien éventuel. Pour cela nous avons procédé, d'une part, à une étude quantitative et biométrique des corps jaunes en relation avec les stades embryonnaires, d'autre part, à une étude histochimique comportant la recherche des lipides figurés et la détection de l'activité $\Delta 5-3 \beta$ hydroxystéroide déshydrogénasique $(3 \beta-H S D H)$. La présence de cette activité enzymatique qui intervient dans la biosynthèse des stéroïdes constitue un argument en faveur du caractère stéoidogène de la structure étudiée.

\section{Matériel et méthodes.}

Nous avons procédé à la confection de coupes sériées de 34 ovaires provenant de femelles gestantes ou ayant mis bas. Ces femelles ont été fixées au liquide de Bouin immédiatement après leur capture. Les coupes ont été colorées par le trichome de Masson-Goldner ou par l'azan de Romeis. Nous avons également procédé à la détection des lipides par le noir Soudan et à la détection de l'activité $3 \beta-H S D H$ sur des ovaires frais provenant de femelles maintenues vivantes pendant un mois au laboratoire, après leur capture.

PLANCHE I

Coupe d'ovaire

FIG. 1, 2, 3. - Vues d'un ovaire (trichrome de Masson-Goldner).

FIG. 4. - Détection des lipides dans un ovocyte en vitellogenèse.

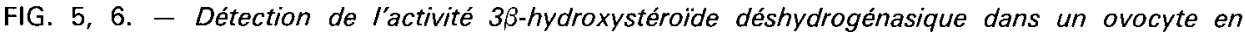
vitellogenèse.

$\mathrm{CJ}=$ corps jaune $; \mathrm{FA}=$ follicule atrétique $; \mathrm{GV}=$ globules vitellins $; \mathrm{gr}=$ granulosa $;$ ov = ovocyte; th $=$ thèque. 

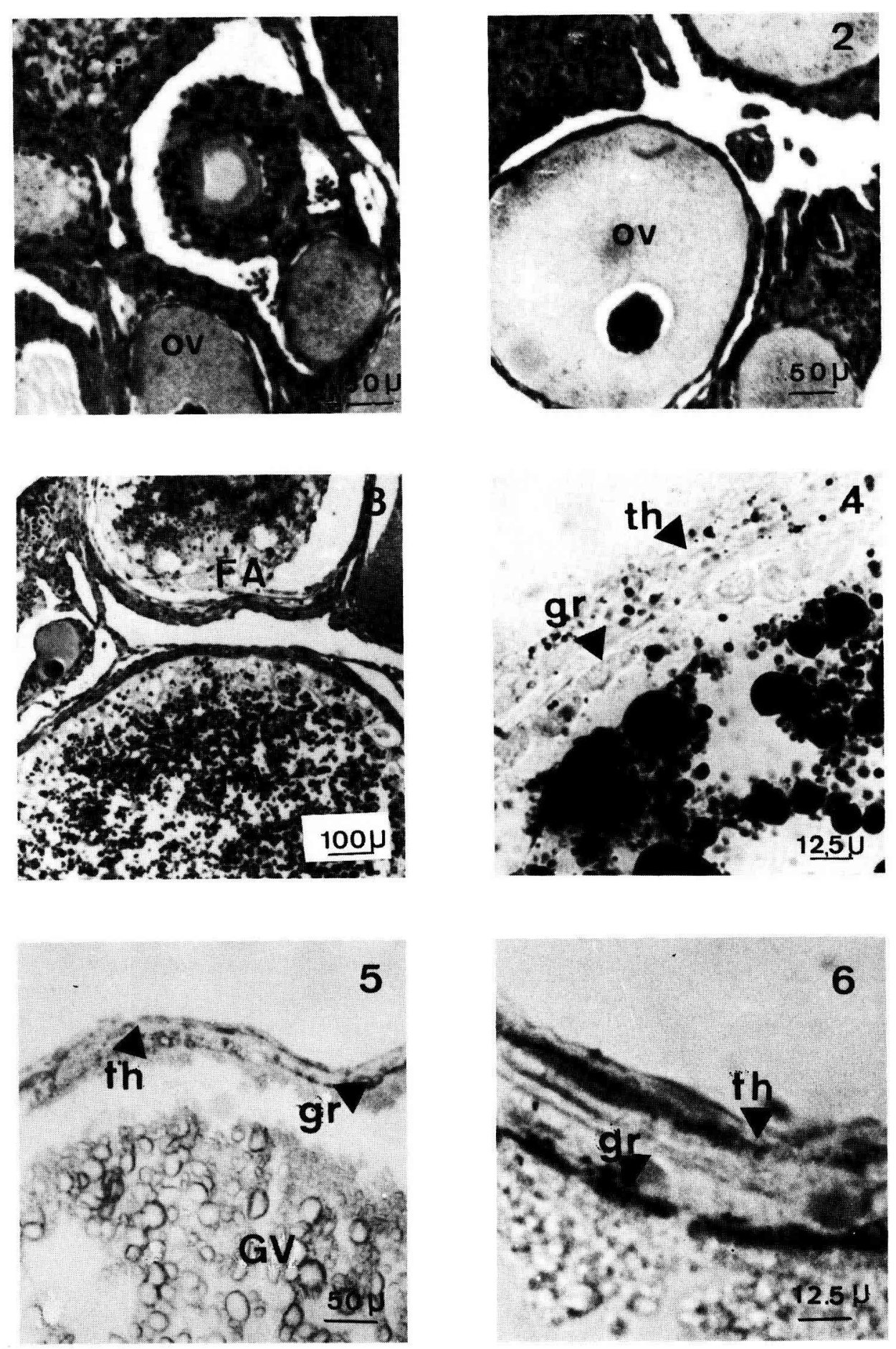
L'activité $3 \beta-H S D H$ a été mise en évidence sur coupes après inclusion dans la paraffine, selon la méthode décrite par Collenot et Collenot (1977). Les fragments d'ovaire ont été fixés de 30 à $60 \mathrm{~min}$ à froid $\left(0\right.$ à $\left.4^{\circ}\right)$ dans une solution tamponnée (tampon phosphate $0,1 \mathrm{M}$ ) de formaldéhyde, préparée extemporanément à partir de paraformaldéhyde. Les pièces ont été débitées en coupe épaisses $(100$ à $150 \mu)$ à l'aide du "Tissue Sectionneur » de Smith et Farquhar. Après un rinçage prolongé (2 à $12 \mathrm{~h}$ ), les coupes ont été incubées dans un milieu comportant $1 \mathrm{mg}$ d'éthiocholane (Sigma), préalablement dissoute dans $0,5 \mathrm{ml}$ de diméthyl-formamide contenant $5 \mathrm{mg}$ de NAD et $5 \mathrm{mg}$ de Nitro BT. Après une incubation de 45 à 60 min à $37^{\circ}$, les pièces sont rincées dans la solution tampon ( 3 bains successifs de $1 \mathrm{~h}$ ), fixées au formol à $10 \%$ pendant $1 \mathrm{~h}$, rincées, puis rapidement déshydratées, éclaircies dans le toluène et imprégnées dans la paraffine. Les noyaux sont colorés par le rouge nucléaire ou le vert de méthyle. Cette méthode fournit des préparations claires permettant une bonne localisation de I'activité enzymatique.

\section{Résultats.}

\section{Histologie et histochimie de l'ovaire avant la ponte ovulaire.}

Chaque ovaire de Typhlonectes compressicaudus comporte un nombre variable de follicules : 20 à 200 par ovaire selon les individus. Ces follicules ont été classés en plusieurs catégories correspondant à des états d'évolution différents. Notre classement est basé sur la taille et la structure histologique du follicule (fig. 1 et 2). En décembre-janvier, juste avant la ponte ovulaire, toutes les catégories folliculaires sont représentées dans les ovaires de toutes les femelles adultes. On a ainsi pu observer:

- quelques follicules de petite taille (diamètre inférieur à $150 \mu$ ) regroupés et formant des zones de prolifération, alors inactives, qui sont dispersées tout au long de l'ovaire ;

— des follicules de 150 à $600 \mu$, dont l'ovocyte possède quelques petites inclusions à la périphérie du cytoplasme; ils représentent $30 \%$ des follicules totaux : — des follicules de 600 à $750 \mu$, plus évolués, avec une thèque conjonctive organisée, une granulosa formée d'une couche cellulaire séparée de l'ovocyte par une mince membrane vitelline ; ils représentent $20 \%$ de l'ensemble des follicules ;

- des follicules de 750 à $1200 \mu$ dont l'ovocyte est en prévitellogenèse et possède un cytoplasme très granuleux, colorable par l'orangé $G$; ces follicules représentent environ $13 \%$ du total ;

- des follicules de 1200 à $2000 \mu$ dont l'ovocyte en vitellogenèse possède un cytoplasme empli de globules vitellins de 5 à $7 \mu$ de diamètre, fortement colorables par l'orangé $\mathrm{G}$; ils représentent $31 \%$ des follicules totaux ;

- Quelques follicules atrétiques représentant $5 \%$ de l'ensemble sont également présents (fig. 3).

La détection histochimique de l'activité $3 \beta-\mathrm{HSDH}$ a permis de montrer que, dès que l'ovocyte est en prévitellogenèse, un dépôt de formazan apparaît au 
niveau de la granulosa et au niveau de la thèque (dans ce dernier cas, il est de faible intensité) (fig. 4). De même, quelque granulations de lipides soudanophiles ont pu être mises en évidence au niveau de ces cellules (fig. 5 et 6).

\section{Histologie, histochimie et évolution des corps jaunes post-ovulatoires}

La moitié environ des femelles adultes subissent la ponte ovulaire. Chez ces femelles, les deux-tiers seulement des ovocytes en vitellogenèse vont être pondus. Les follicules correspondant aux ovocytes pondus vont alors évoluer en corps jaunes post-ovulatoires qui représenteront 14 à $17 \%$ des follicules (voir tabl. 1). Les ovocytes non pondus dégénèrent et leurs follicules deviennent atrétiques.

TABLEAU 1

Evolution du nombre et de la structure des corps jaunes au cours du cycle annuel.

\begin{tabular}{ccccc}
\hline $\begin{array}{c}\text { Stade } \\
\text { embryonnaire }\end{array}$ & $\begin{array}{c}\text { Nombre de corps } \\
\text { jaunes (en \% de } \\
\text { follicule) }\end{array}$ & $\begin{array}{c}\text { Diamètre moyen } \\
\text { des corps jaunes } \\
\text { (en } \mu)\end{array}$ & $\begin{array}{c}\text { Nombre de corps } \\
\text { jaunes cavitaires } \\
\text { par rapport au } \\
\text { nombre total }(\%)\end{array}$ & $\begin{array}{c}\text { Surface de la } \\
\text { cavité len \% de } \\
\text { la surface de } \\
\text { coupe) }\end{array}$ \\
\hline I et II & $14-15$ & 1600 & 100 & 29 \\
\hline III & $14-15$ & 1500 & 58 & 23 \\
\hline IV & 17 & 1200 & 3 & 2 à 9 \\
\hline Nouveau-nés & 11 & 600 & 0 & \\
\hline
\end{tabular}

Nous avons étudié l'évolution des corps jaunes au cours de la gestation en fonction des stades embryonnaires déterminés selon Exbrayat, Delsol et Flatin (1981) et Delsol et al. (1981).

Au début de la gestation, alors que les embryons sont aux stades I ou II (premiers stades de développement ou embryons avec une vésicule vitelline entourés d'une ganguel, chaque corps jaune mesure en moyenne $1600 \mu$. Les cellules folliculaires se sont multipliées et ont changé d'aspect. Elles sont sphériques ( $30 \mu$ de diamètre environ) ou allongées $(20 \times 40 \mu)$, avec un cytoplasme granuleux et un noyau de $10 \mu$ de diamètre rejeté vers la périphérie. Ces cellules délimitent une cavité centrale importante qui représente $30 \%$ de la surface de la coupe centrale d'un corps jaune (fig. 7). Cette cavité est emplie d'une sécrétion colorée en bleu ou gris-bleu par l'Azan de Romeis. Nous avons constaté que très peu de cellules seulement sont rejetées dans la cavité. La vascularisation est essentiellement localisée à la périphérie, au niveau de la thèque conjonctive qui présente plusieurs couches de cellules.

Lorsque les embryons atteignent les stades III, en mai-juin (ils sont alors démunis d'enveloppe et leur vésicule vitelline est résorbée), les corps jaunes 
mesurent encore $1500 \mu$ en moyenne. $58 \%$ d'entre eux seulement possèdent encore une cavité représentant $23 \%$ de la surface de la coupe centrale. Chez les autres, les cellules ont envahi la cavité pour donner un tissu compact (fig. 8), comme chez Nectophrynoides occidentalis (Lamotte, Rey et Vogeli, 1964). Cependant, chez Typhlonectes compressicaudus, des capillaires sanguins sont visibles entre les cellules du corps jaune.

Dès juillet, chez les femelles portant des embryons de stade IV (larves), le volume des corps jaunes a diminué (le diamètre est de $1200 \mu$ environ). La cavité centrale de chacun d'eux est complètement envahie de cellules; certaines d'entre elles présentent des vacuoles au niveau du cytoplasme. Cependant, dans quelques corps jaunes, une petite cavité représentant 2 à $9 \%$ de la surface de coupe est encore perceptible. De nombreux éléments sanguins sont présents dans les capillaires.

Enfin, chez les femelles qui viennent de mettre bas, les corps jaunes ont fortement diminué de volume. Ils ne mesurent plus que $600 \mu$ de diamètre, en moyenne. Leur cavité n'existe plus. Les cellules deviennent toutes très allongées, leur noyau entrant en pycnose ; de nombreux éléments sanguins sont toujours présents. Les corps jaunes dégénèrent rapidement en laissant des traces qui se confondent avec le tissu conjonctif (fig. 2). Les corps jaunes encore reconnaissables ne représentent plus que $11 \%$ des follicules totaux.

La détection de l'activité $3 \beta-\mathrm{HSDH}$ met en évidence un dépôt de formazan important au niveau des cellules du corps jaune, cavitaire ou non (fig. 10). $D^{\prime}$ autre part, ces cellules présentent une réaction fortement positive à la détection des lipides par le noir Soudan (fig. 9).

\section{Histologie, histochimie et évolution des follicules atrétiques}

Les ovocytes en vitellogenèse non pondus donnent lieu à la formation de follicules atrétiques (fig. 3 et 11). Les plus gros (plus de $1200 \mu$ de diamètre) présentent une invasion cellulaire périphérique. Les globules vitellins paraissent alors phagocytés de façon centripète. Au fur et à mesure que l'atrésie se poursuit, le follicule diminue de taille et s'emplit de cellules disposées irrégulièrement. En fin

\section{PLANCHE II}

FIG. 7. - Corps jaune cavitaire, en début de gestation.

FIG. 8. - Corps jaune, en fin de gestation.

FIG. 9. - Détection des lipides dans un corps jaune.

FIG. 10. - Détection de l'activité 3ß-hydroxystéroïde déshydrogénasique dans un corps jaune.

FIG. 11. - Follicule atrétique.

FIG. 12. - Détection des lipides dans un follicule atrétique (même légende que dans la planche I). 

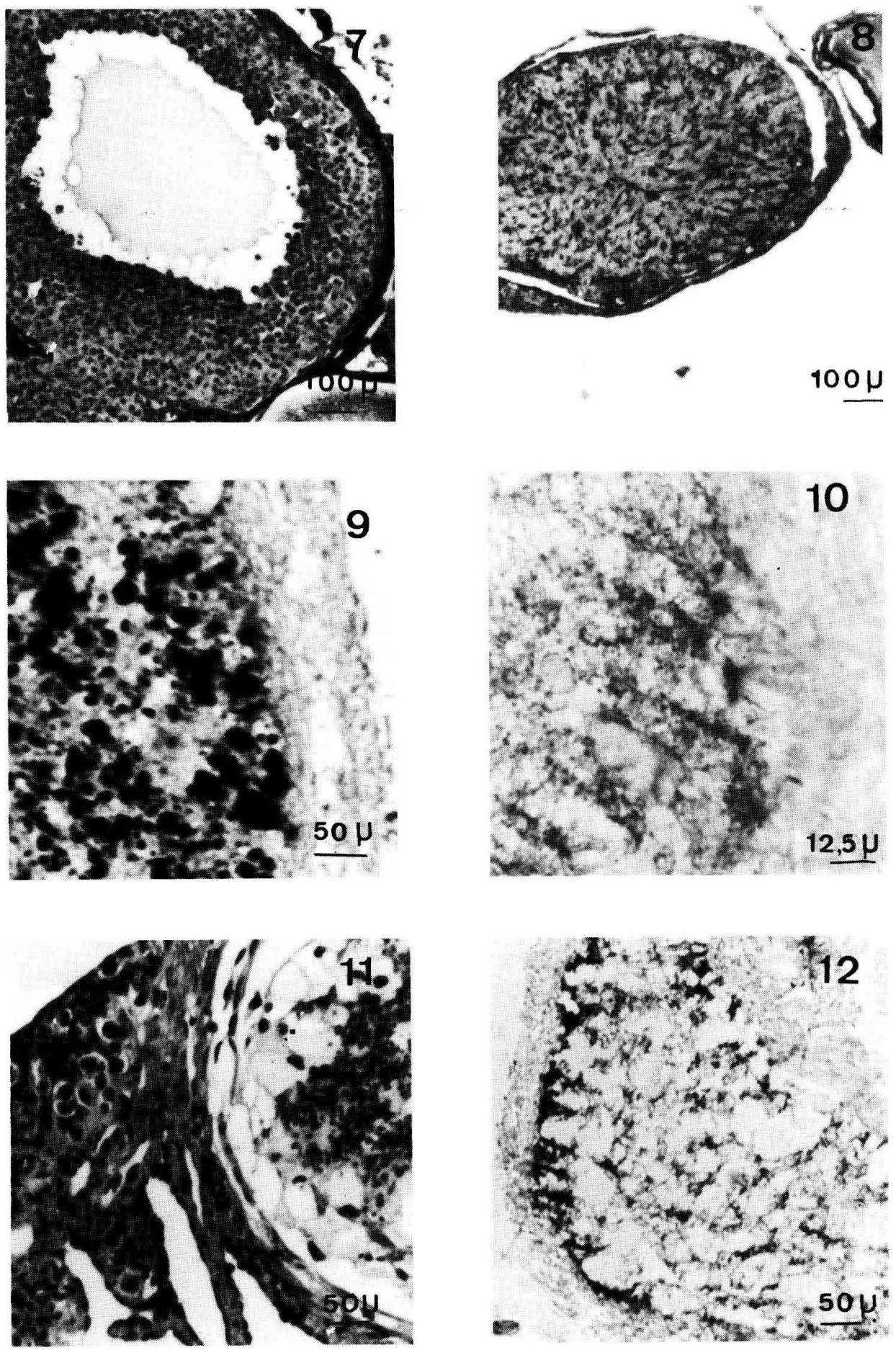
d'atrésie, de tout petits follicules de moins de $600 \mu$ de diamètre sont emplis d'un tissu dense à cellules allongées au noyau pycnotique et disposées de manière anarchique. Ces structures, comme les corps jaunes dégénérés, finissent par se confondre avec le tissu conjonctif.

Aucune activité de la $\Delta$ 5-3 $\beta$-hydroxystéroïde déshydrogénase n'a pu être détectée. Par contre, on note la présence de granulations lipidiques soudanophiles (fig. 12).

4. Relations entre le nombre d'ovocytes en vitellogenèse, le nombre de corps jaunes et le nombre d'embryons.

Chez Typhlonectes compressicaudus, la vitellogenèse s'effectue entre octobre et décembre. En janvier, au moment de la ponte ovulaire, une vingtaine d'ovocytes en vitellogenèse sont prêts à être utilisés : ils représentent de 30 à $40 \%$ de l'ensemble des follicules dont le diamètre est supérieur à $150 \mu$. A côté de ces derniers, $15 \%$ environ des follicules sont en prévitellogenèse.

Les deux tiers environ seulement des ovocytes en vitellogenèse seront pondus et les follicules restants évolueront en corps jaunes. Tous les follicules dont les ovocytes sont en vitellogenèse, ainsi que ceux dont les ovocytes sont en prévitellogenèse deviennent atrétiques.

Si l'on compare le nombre de corps jaunes par ovaire et le nombre d'embryons portés par l'utérus correspondant, il apparaît une différence considérable entre ces deux nombres: au début de la gestation, sur une vingtaine d'ovocytes pondus, 4 à 6 embryons seulement pourront se développer ; au fur et à mesure que la gestation se poursuit, le nombre d'embryons diminue encore (2 ou 3 par utérus à la fin de la gestation).

Enfin, les résultats montrent que le nombre moyen de corps jaunes len pourcentage de follicules) est plus faible après la mise bas.

\section{Discussion et conclusion.}

Typhlonectes compressicaudus est un Amphibien Apode vivipare. Chez les femelles gestantes, au moment de la reproduction, les ovaires présentent des structures qui rappellent, par leur évolution et par certains caractères histochimiques, les corps jaunes observés chez d'autres Amphibiens Apodes (Wake, 1968, 1977), chez des Urodèles ovovivipares ou vivipares (Salamandra atra : Vilter et Vilter, 1960, 1964 ; Salamandra salamandra : Joly et Picheral, 1972) ou chez des Anoures vivipares (Nectophrynoides occidentalis : Lamotte, Rey et Vogeli, 1964 ; Xavier, Zuber-Vogeli et Le Quang Trong, 1970).

Les corps jaunes de Typhlonectes compressicaudus rappellent ceux de $\mathrm{Nec}$ tophrynoides occidentalis (bien que ces derniers se soient pas vascularisés). Leur évolution et similaire : ils sont cavitaires au début de la gestation ; puis la cavité est rapidement oblitérée par les cellules de la granulosa, alors que le volume total du follicule diminue. Le corps jaune de Typh/onectes persiste durant toute la 
période de gestation; à la parturition, il dégénère rapidement, plus rapidement, semble-t-il, que celui de Nectophrynoides.

Chez Nectophrynoides, Lamotte, Rey et Vogeli (1964) ont montré que le nombre total de corps jaunes est égal au nombre d'embryons, alors que Vilter et Vilter (1960) avait montré que chez Salamandre atra il y avait environ vingt corps jaunes pour un embryon. Le cas de Typhlonectes ressemble à celui de Salamandra atra car en définitive sur une vingtaine d'ovocytes pondus seulement quatre à six se développeront en embryons qui ne seront pas tous viables (Delsol et al., 1981 ; Exbrayat, Delsol et Flatin, 1981).

I'étude de l'activité $3 \beta-\mathrm{HSDH}$ et des lipides figurés donne des résultats comparables à ceux qui ont été trouvés chez Salamandra salamandra (Joly et Picheral, 1972) ou chez Nectophrynoides occidentalis (Xavier, Zuber-Vogeli et Le Quang Trong, 1970).

Les ovocytes en vitellogenèse sont entourés d'une granulosa présentant une réaction $3 \beta-H S D H$ positive et d'une thèque également positive, mais à un degré moindre. Après la ponte ovulaire, seules les cellules du corps jaune let non plus celles de la thèque) présentent une telle réactivité. Les follicules atrétiques, issus de la dégénérescence des ovocytes non pondus, sont - comme chez les deux espèces précédemment citées - exempts de toute activité enzymatique.

Il est vraisemblale que - comme chez les autres Amphibiens vivipares ou ovovivipares - la granulosa et la thèque des ovocytes en prévitellogenèse ou en vitellogenèse, ainsi que les cellules des corps jaunes, secrètent des hormones stéroïdes. Des études ultérieures permettront de préciser ce point.

Quant au rôle physiologique des corps jaunes, il est vraisemblable que celuici se manifeste dans les importantes modifications que subissent les oviductes avant et pendant la gestation (Exbrayat, Delsol et Flatin, 1982).

Après la période de mise bas (octobre), la vitellogenèse reprend chez la plupart des femelles adultes. Parmi elles, $50 \%$ environ n'ont pas été en gestation et les autres viennent de mettre bas (Exbrayat, 1983). Chez ces dernières, nous observons un accroissement rapide des follicules de 600 à $750 \mu$. La reprise immédiate de la vitellogenèse chez ces mêmes animaux pourrait être en relation avec la disparition de l'activité hormonale des corps jaunes. Notons que chez un autre Amphibien Apode vivipare, Dermophis mexicanus, Wake (1980) n'observe aucune vitellogenèse chez les femelles qui viennent de mettre bas. Cependant cette espèce a un développement embryonnaire plus lent que celui de Typhlonectes compressicaudus et, lorsque les jeunes naissent, la période de vitellogenèse est dépassée.

II est à noter également que le nombre de petits ovocytes de 150 à $600 \mu$ augmente en avril chez les femelles gestantes ou non : ceci pourrait suggérer que, chez les premières, l'effet de blocage des hormones des corps jaunes ne se fait pas ressentir à ce stade de l'ovogenèse. Il est à préciser que, à cette période, les corps jaunes sont encore au début de leur évolution. 


\section{Références}

COLLENOT G., COLLENOT A., 1977. L'activité $3 \beta$-hydroxystéroïde déshydrogénasique dans les gonades en différenciation de Pleurodeles waltlii (Amphibien Urodèle); visualisation sur coupes sériées à l'aide d'une nouvelle technique histochimique. J. Embryol. exp. Morph., 42, 29-42.

DELSOL M., FLATIN J., EXBRAYAT J.-M., BONS J. 1981. - Développement de Typhlonectes complessicaudus, Amphibien Apode vivipare. Hypothèse sur sa nutrition embryonnaire et larvaire par un ectotrophoblaste. C. R. Acad. Sci., Paris, Sér. III, 293, 281-285.

EXBRAYAT J. M., 1983 Premières observations sur le cycle annuel de l'ovaire de Typhlonectes compressicaudus (Duméril et Bibron, 1841), Batracien Apode vivipare. C. R. Acad. Sci., Paris, Sér. III, 296, 493-498.

EXBRAYAT J. M., DELSOL M., FLATIN J., 1981. Premières remarques sur la gestation chez Typhlonectes compressicaudus (Duméril et Bibron, 1841), Amphibien Apode vivipare. C. R. Acad. Sci., Paris, Sér. III, 292, 417-420.

EXBRAYAT J. M., DELSOL M., FLATIN J., 1982. Observations concernant la gestation de Tryphlonectes compressicaudus (Duméril et Bibron, 1841\}, Amphibien Apode vivipare. Bull. Soc. Zool. Fr., 107, 486.

EXBRAYAT J. M., SENTIS Ph., 1982. Homogénéité du testicule et cycle annuel chez Typh/onectes compressicaudus (Duméril et Bibron, 1841), Amphibien Apode vivipare. $C$. $R$. Acad. Sci., Paris, Sér. III, 294, 757-762.

JOLY J., 1964. La présence de lipides biréfringents dans les corps jaunes post-ovulatoires de la Salamande tachetée. C. R. Acad. Sci, Paris, 258, 3563-3565.

JOLY J., 1965. Mise en évidence histochimique d'une $\triangle 5$-3- $\beta$-hydroxy-stéroïde-déshydrogénase dans l'ovaire de l'Urodèle Samamandra salamandra (L.) à différents stades du cycle sexuel. C. R. Acad. Sci., Paris, 261, 1569-1571.

JOLY J., PICHERAL B., 1972. Ultrastructure, histochimie et physiologie du follicule pré-ovulatoire et du corps jaune de I'Urodèle ovo-vivipare Salamandra salamandra (L.). Gen. comp. Endocrinol., 18, 235-259.

LAMOTTE M., REY P., 1954. Existence de corpora lutea chez un Batracien Anoure vivipare, Nectophrynoides occidentalis Angel; leur évolution morphologique. C. R. Acad. Sci., Paris, 238, 393-395.

LAMOTTE M., REY P., VOGELI M., 1964. Recherches sur l'ovaire de Nectophrynoides occientalis Angel (Amphibien Anoure vivipare). II - Synthèse in vitro des stéroïdes.

OZON R. XAVIER F., 1968. Biosynthèse in vitro des stéroïdes par l'ovaire de l'Anoure vivipare Nectophrynoides occidentalis au cours du cycle sexuel. C. R. Acad. Sci. Paris, Sér. D, 266. 1173-1175.

VILTER V., VILTER A., 1960. Sur la gestation de la Salamandre noire des Alpes, Salamandra atra Laur. C. R. Soc. Biol., 154, 290-294.

VILTER V., VILTER A., 1964. Sur l'évolution des corps jaunes ovariens chez Salamandra atra Laur. des Alpes vaudoises. C. R. Soc. Biol., 158, 457-461.

WAKE M. H., 1968. Evolutionary morphology of the Caecilian urogenital system. I - The gonads and the fat bodies. J. Morphol., 126, 291-232.

WAKE M. H., 1977. The reproductive biology of Caecilians: an evolutionary perspective, 73-100. In D. H. TAYLOR, S. I. GUTTMANN, The reproductive biology of amphibians, Miami Univ. Oxford, Ohio.

WAKE M. H., 1980. Reproduction, growth and population structure of the Central American Caecilian Dermophis mexicanus. Herpetologica, 36, 244-256.

XAVIER F., OZON R., 1971. Recherches sur l'activité endocrine de l'ovaire de Nectophrynoides occidentalis Angel Amphibien Anoure vivipare). II - Synthèse in vitro des stéroïdes. Gen. comp. Endocrinol., 16, 30-40.

XAVIER F., ZUBER-VOGELI M., LE QUANG TRONG Y., 1970. Recherches sur I'activité endocrine de l'ovaire de Nectophrynoides occidentalis Angel (Amphibien Anoure vivipare). 1 - Etude histochimique. Gen. comp. Endocrinol., 15, 425-431. 